Pacific Journal of Mathematics

HOLLOW MODULES AND LOCAL ENDOMORPHISM RINGS 


\section{HOLLOW MODULES AND LOCAL ENDOMORPHISM RINGS}

\section{PATRICK FLEURY}

This is a study of the conditions under which smallness of proper submodules will influence the structure of the endomorphism ring of a module. The case in which that endomorphism ring becomes local is of special interest. Further facts about hollow modules are also established.

1. Introduction. Throughout this paper, $R$ will denote an associative ring with unit and $R$-Mod the category of left $R$-modules. In [3], we studied elements of $R$-Mod such that every strictly decreasing sequence of submodules eventually became small. Such modules were said to have finite spanning dimension and we showed that each such could be represented as the sum of a unique number of hollow submodules. A module was said to be hollow if every submodule were small. That is, $X$ is hollow if $X, Y_{1}, Y_{2} \in R$-Mod, $Y_{1}, Y_{2} \leqq X$, and $Y_{1}+Y_{2}=X$ imply either $Y_{1}=X$ or $Y_{2}=X$. In this paper, we present some theorems about hollow modules and, in particular, we investigate the circumstances under which certain hollow modules have local endomorphism rings. By a local ring we simply mean a ring with a unique maximal left ideal and we assume neither chain conditions nor commutativity.

To a certain extent, hollow modules play the same role for modules with finite spanning dimension which simple modules play for modules with descending chain condition. For example, theorems about hollow modules having local endomorphism rings are extensions of Shur's lemma.

In $\S 2$, we establish basic facts about hollow modules and in $\S 3$ we introduce the idealizer and show its relation to endomorphism rings. In $\S 4$, we present eight theorems which show that, under certain conditions, a hollow module has a local endomorphism ring. In $\S 5$, we present some miscellaneous results on when a maximal left ideal is 2-sided and we show that the abelian group of $p$-adic integers is hollow.

2. Some preliminaries. We recall that if $X$ is an element of $R$-Mod and $Y_{1}$ is a submodule of $X$, then $Y_{1}$ is called small if $Y_{1}+$ $Y_{2}=X$ for some submodule $Y_{2}$ of $X$ implies $Y_{2}=X$. It is easy to show that the image of a small submodule is again small and we will have use for this fact later. 
Proposition 2.1. If $X$ is a hollow left $R$-module which is also finitely generated, then $X$ is cyclic. Furthermore, if $X \cong R / I$, then $I$ is contained in a unique maximal left ideal $M$. Conversely, if $I$ is contained in a unique maximal left ideal, then $R / I$ is hollow.

Proof. Suppose $x_{1}, \cdots, x_{n}$ form a set of generators for $X$. Then $X=R x_{1}+\cdots+R x_{n}$. Since every proper submodule of $X$ is small, we may delete the summands one by one until we find $X=R x_{\imath}$ for some $i$. Thus $X$ is cyclic.

We now have $X \cong R / I$ where $I$ is the annihilator of $x_{i}$. Suppose $I$ is contained in two maximal ideals, say $M_{1}$ and $M_{2}$. Then $M_{1}+$ $M_{2}=R$ so the sum of the images of $M_{1}$ and $M_{2}$ in $R / I$ actually equal $R / I$. But it is clear that $M_{1} / I$ and $M_{2} / I$ are both proper submodules of $R / I$ and so we have a contradiction. Thus $I$ is contained in a unique maximal left ideal.

Finally, suppose $I$ is contained uniquely in the maximal left ideal $M$. Then any submodule of $R / I$ corresponds to a left ideal of $R$ containing $I$. All such proper ideals must be contained in $M$ so any finite sum of proper submodules of $R / I$ is contained in $M / I$.

CoRollary 2.2. If $X$ is hollow and finitely generated, then $X$ contains a unique maximal submodule.

Proof. We know from the theorem that $X \cong R / I$ where $I$ is contained in a unique maximal left ideal, $M$, of $R$. So any proper submodule of $R / I$ must be contained in $M / I$ which is the unique maximal submodule of $R / I$.

Hidden in these proofs is the germ of a useful idea. We can dualize a notion of Goldie in the following way. Let $X \in R$-Mod and let $Y_{1}, Y_{2}$ be submodules of $X$. We say that $Y_{1}$ and $Y_{2}$ are correlated if $Y_{1}+H=X$ if and only if $Y_{2}+H=X$ for some submodule, $H$, of $X$. It is very easy to see that correlation is an equivalence relation. Furthermore, if $R / I$ is hollow, then $I$ must be correlated to the maximal left ideal $M$ which contains it in $R$; for if $M$ is a maximal left ideal which contains $R$ then $I+H=R$ certainly implies $M+$ $H=R$. On the other hand, if $M+H=R$, then, taking the canonical images of $M$ and $H$ in $R / I$, we have $M / I+(H+I) / I=R / I$. Since $M / I$ is small in $R / I$ we see that $(H+I) / I=R / I$ or $H+I=R$.

Conversely, if $I$ is correlated to $M$, then $I$ must be contained uniquely in $M$. This is because the containment of $I$ in another maximal left ideal $M^{\prime}$ would imply $I+M^{\prime}=R$ from the relationship $M+M^{\prime}=R$. But since $I \subseteq M^{\prime}, I+M^{\prime}=M^{\prime}$. We thus have Theorem 2.3 which is stated below in an equivalent but more useful form. 
TheOREM 2.3. Let $I$ be a left ideal of $R$. Then $I$ is contained in a unique maximal left ideal $M$ if and only if $R x+I=R$ for all $x \in R$ and $x \notin M$.

The following propositions show that there are plenty of left ideals which are contained uniquely in maximal ideals.

Proposition 2.4. If $I$ is a left ideal of $R$ and is contained uniquely in the maximal left ideal $M$, then $I^{2}$ is contained uniquely in $M$.

Proof. Suppose $I^{2}$ is contained in the maximal left ideal $M^{\prime} \neq M$. Then there is $m_{1} \in M^{\prime}, m_{1} \notin M$, so $R m_{1}+I=R$. Thus there is $r \in R$, $i \in I$ and $r m_{1}+i=1$. Let $i^{\prime}$ be any element of $I$. Then $i^{\prime} r m_{1}+$ $i^{\prime} i=i^{\prime}$. Both the first and second terms in the sum are in $M^{\prime}$. Thus $I \subseteq M^{\prime}$ which is a contradiction.

CoRollary 2.5. If $I$ is a left ideal of $R$ which is contained uniquely in a maximal left ideal, $M$, then $I^{n}$ is contained uniquely in $M$ for all $n \geqq 1$.

CoROllary 2.6. If $M$ is a maximal left ideal of $R$, then $M^{n}$ is contained uniquely in $M$ for all $n \geqq 1$.

CoRollary 2.7. If $I$ is a left ideal of $R$ which contains some power of the maximal left ideal $M$, then $I$ is contained uniquely in $M$.

CoRollary 2.8. If $I$ is a nilpotent left ideal of $R$ which is contained uniquely in a maximal left ideal $M$, then $R$ is local.

3. The idealizer. From now on, we will assume that all modules are hollow and cyclic unless otherwise stated. We shall be interested in $\operatorname{Hom}_{R}(R / I, R / I)=\operatorname{End}_{R}(R / I)$ where $R / I$ is hollow. Each $R$ homomorphism $f: R / I \rightarrow R / I$ is uniquely determined by its image on $1+I$. Thus $f(1+I)=a+1$, so $f(r+I)=r(f(1+I)=r a+I$ and $f$ corresponds to $a+I$. Furthermore, $f(0+I)=0+I$ so if we choose any other representative for zero, say $i+I$, then $f(i+I)=i a+I=$ $0+I$. That is, $i a \in I$. We then set $\mathscr{I}(I)=\{x \in R: I x \subseteq I\} . \mathscr{I}(I)$ is called the idealizer of $I$ and $\operatorname{End}_{R}(R / I)$ is obviously isomorphic to $\mathscr{J}(I) / I$. (Cohn, [1], Proposition 4.1, p. 17).

Since every $R$-endomorphism of $R / I$ corresponds to an element of $\mathscr{I}(I) / I$, we may think of an $R$-endomorphism as right multiplication of $R / I$ an element of $R$. This observation will be useful later. 
Proposition 3.1. If the left ideal $I$ of $R$ is contained uniquely in the maximal left ideal $M$, then $\mathscr{I}(I) \subseteq \mathscr{J}(M)$.

Proof. Let $r \in \mathscr{F}(I)$. Thus $I r \subseteq I$. Now $r$ induces an $R$-endomorphism of $R / I$ by sending $a+I$ to $a r+I$. We know the image of a small submodule under an $R$-homomorphism is again small. Thus the image of $M / I$ is small in $R / I$ so it must be contained in $M / I$. Thus if $m \in M, m r+I \in M / I$ which, in turn, implies $m r \in M$.

CoRollary 3.2. If $I$ is an ideal of $R$ and $I$ is contained uniquely in the maximal left ideal $M$, then $M$ is an ideal.

Proof. We know that $R \supseteqq \mathscr{I}(M) \supseteqq \mathscr{I}(I)=R$. Thus $\mathscr{I}(M)=R$ so $M$ is an ideal.

Proposition 3.3. Let $R / I$ be hollow and $x \in \mathscr{J}(I)$. Then the endomorphism induced by $x$ on $R / I$ is epic if and only if $x \notin M$ where $M$ is the unique maximal left ideal containing $I$.

Proof. The endomorphism induced by $x$ is epic if and only if $R / I=(R x+I) / I$. But that is true if and only if $R x+I=R$ which is true if and only if $x \notin M$.

4. Local endomorphism rings. With the help of the foregoing theorems, it is now possible to ask and answer some questions concerning whether or not $\operatorname{End}_{R}(R / I)$ is local when $R / I$ is hollow. The obvious conjectures and the main difficulties may be described as follows. We know that $\operatorname{End}_{R}(R / I)$ is isomorphic to $\mathscr{J}(I) / I$. We would thus expect that if $\operatorname{End}_{R}(R / I)$ is a local ring, its unique maximal ideal would be $M \cap \mathscr{J}(I) / I$ where $M$ is the unique maximal left ideal containing $I$. This is indeed so in the cases we will consider. In the general case, however, the following difficulty arises. Suppsse $a \in \mathscr{I}(I)$ and $a \notin M$. We would like to show that $a$ induces an isomorphism on $R / I$. The obvious proof to try is as follows. Since $a \notin M$, we know $R a+I=R$. Thus there is $r \in R$ and $r a+i=1$. We would then like to say that $r$ induces an inverse map for $a$ on $R / I$. Unfortunately, we also need to know that $I r \subseteq I$ and possibly this is not so. Thus there is a possibility that $\operatorname{End}_{R}(R / I)$ is not local and we must specialize.

The following observations are also useful. Suppose $a \in \mathscr{F}(I)$, $a \notin M$. Then there is $r \in R$ and $r a+i_{1}=1$ for some $i_{1}$. By Proposition 3.1, $r \notin M$ since, otherwise $1 \in M$. Thus $R r+1=R$ so there is $x \in R$ with $x r+i_{2}=1$. We then have $x r a+x i_{1}=x$ and $x r a+$ $i_{2} a=a$. Thus $x=a-i_{2} a+x i_{1}=a+x i_{3}$. Thus $x \in \mathscr{I}(I)$ and induces 
the same endomorphism on $R / I$ that $a$ does.

THEOREM 4.1. If $R / I$ is hollow and $I$ is an ideal, then $\operatorname{End}_{R}(R / I)$ is local.

Proof. It is easy to see that $\operatorname{End}_{R}(R / I) \cong R / I$ is a ring and that $R / I$ has $M / I$ as its unique maximal ideal where $M$ is the unique maximal ideal containing $I$.

We recall that, if $a \in R,(I: a)=\{x \in R \mid x a \in I\}$ is a left ideal. If $a \in \mathscr{I}(I)$, then $(I: a)$ contains $I$.

THEOREM 4.2. Let $I$ be a left ideal of $R$ which is contained uniquely in the maximal left ideal $M$ of $R$. Suppose $R$ satisfies the following chain condition. For every $a \in \mathscr{I}(I)$, the sequence of left ideals

$$
I \subseteq(I: a) \subseteq\left(I: a^{2}\right) \subseteq \cdots
$$

eventually stabilizes. Then $\operatorname{End}_{R}(R / I)$ is local.

Proof. If $M$ is a left $R$-module and $f: M \rightarrow M$ is an epimorphism such that the sequence of submodules

$$
\text { ker } f \subseteq \operatorname{ker} f^{2} \subseteq \cdots
$$

eventually stabilizes, then a standard argument shows that $f$ is an isomorphism. Moreover, it is easy to see that, if $M$ is a hollow module with the property that every epimorphism from $M$ to $M$ is an isomorphism, then $\operatorname{End}_{R}(M)$ is local. We need only note that, in that case, nonunits would form an ideal. Thus it is enough to show that, for any endomorphism $f: R / I \rightarrow R / I$, the above sequence becomes stable. If $f \in \operatorname{End}_{R}(R / I)$, then there exists $a \in \mathscr{I}(I)$ such that $f(r+I)=$ $r a+I$ for all $r+I \in R / I$. Then for any $n \geqq 1, \operatorname{Ker} f^{n}=\left(I: a^{n}\right) / I$ and since the sequence

$$
(I: a) \subseteq\left(I: a^{2}\right) \subseteq \cdots
$$

stabilizes, the proof is complete.

We note that if $M$ is any indecomposable $R$-module such that $\operatorname{End}_{R}(M)$ is semi-perfect (in particular, an artinian ring), then $\operatorname{End}_{R}(M)$ is local (see [4], p. 76, Corollary 1). Since any hollow module is indecomposable, the above also holds true for hollow modules.

TheOREm 4.4. Let $R / I$ be hollow. If $R / I$ is either projective or injective, then $\operatorname{End}_{R}(R / I)$ is local.

Proof. The first part follows from Theorem 4.2 of Ware [7]. The second part follows since it is well-known that an indecomposable injective has a local ring of endomorphisms. 
THEOREM 4.5. If $I$ is divisible as a right $\mathscr{J}(I)$ module, and $R / I$ is hollow, then $\operatorname{End}_{R}(R / I)$ is local.

Proof. Let $a$ represent a map from $R / I$ to $R / I$ and $a \notin M$. We would like to show that the map induced by $a$ has an inverse. Since $a \notin M$, there is $r \in R, i_{1} \in I$ and $r a+i_{1}=1$. By the remarks preceding Theorem 4.1, we may as well assume there is $i_{2} \in I$ and $r a+i_{2}=1$. If $I r \subseteq I$, we are done since then $r$ would induce the required inverse for $a$. Now if $i r \in I r$, then $i=i_{3} a$ since $I$ is divisible in its idealizer. So $i r=i_{3} a r=i_{3}-i_{3} i_{2}$ which implies $I r \leqq I$.

Corollary 4.6. If $I$ is injective in its idealizer and $R / I$ is hollow, then $\operatorname{End}_{R}(R / I)$ is local.

THEOREM 4.7. If $I \cap R a=I a$ for all $a \in \mathscr{F}(I)$ and $R / I$ is hollow, then $\operatorname{End}_{R}(R / I)$ is local.

Proof. Let $a \notin M$ induce a map from $R / I$ to $R / I$. We know the map induced by $a$ is epic. If $x a \in I$, then $x a=i_{1} a$ by hypothesis. Thus $\left(x-i_{1}\right) a=0$. From the remarks preceding Theorem 4.1, we can assume there is $r$ with $a r=1-i_{2}, i_{2} I$. Thus $\left(x-i_{1}\right) a r=0=$ $\left(x-i_{1}\right)\left(1-i_{2}\right)$. So $x=i_{1}\left(1-i_{2}\right)+x i_{2} \in I$.

5. Final remarks. Proposition 3.1 and Corollary 3.2 are interesting because they give relationships between the idealization of $I$ and the idealization of the unique maximal ideal containing $I$. There are more theorems like this which relate $I, M$, and the left annihilator of $I$.

Proposition 5.1. Let $I$ be a left ideal of $R$ which is uniquely contained in the maximal left ideal $M$. If either I contains no idempotents or $I^{2} \neq I$, then $(0: I) \subseteq M$.

Proof. Suppose $(0: I) \nsubseteq M$. Then there is $\alpha \in(0: I)$ and $i \in I$ and $\alpha+i=1$ by Theorem 2.3. Thus $i=(\alpha+i) i=\alpha i+i^{2}=i^{2}$. Thus $i$ contains an idempotent. Furthermore, if $i_{1} \in I$, then $i_{1}=$ $(\alpha+i) i_{1}=i i_{1}$ so $i_{1} \in I^{2}$. Thus $I=I^{2}$.

Proposition 5.2. Let $I, M$, and $R$ be as above. Then, if $M$ is not an ideal, $I R=R$ and $(0: I)=0$.

Proof. The ideal $I R$ contains $I$ so either it is contained in $M$ or it is equal to $R$. By Proposition 3.1, if $I R \subseteq M$, then $\mathscr{J}(I R) \subseteq$ $\mathscr{J}(M)$. But $\mathscr{J}(I R)=R$ and $\mathscr{J}(M) \neq R$ by hypothesis. Thus $I R=R$. 
Now suppose $\alpha \in(0: I)$. Since $I R=R$, there are elements $i_{1}, \cdots, i_{n} \in$ $I, r_{1}, \cdots, r_{n} \in R$ and $i_{1} r_{1}+\cdots+i_{n} r_{n}=1$. So $\alpha=\alpha i_{1} r_{1}+\cdots+\alpha i_{n} r_{n}=0$. Thus $(0: I)=0$.

Finally, we will close by showing that some hollow modules are not cyclic. For example, if $p \in Z$ is a prime, consider $Z_{(p)}$ the abelian group of $p$-adic integers. We can show this is hollow in the following way. Suppose $Z_{(p)}=A_{1}+A_{2}$ where $A_{1}$ and $A_{2}$ are both proper subgroups of $Z_{(p)}$. Let $\pi_{n}$ be the canonical projection of $Z_{(p)}$ on $Z / p^{n} Z$. We know each $Z / p^{n} Z$ is hollow. Thus, since $Z / p Z=\pi_{n} A_{1}+$ $\pi_{n} A_{2}$ we must have one, say $\pi_{n} A_{1}$, equal to $Z / p^{n} Z$. But this would imply $\pi_{i} A_{1}=Z / p^{i} Z$ for all $i<n$. Thus $\pi_{n} A_{1}=Z / p^{n} Z$ for all $n$. But then $A_{1}=Z_{(p)}$ and we are done.

As another, and easier, example of an infinitely generated hollow module, we can consider the abelian group $Z\left(p^{\infty}\right)$ for any prime $p$. This is an infinite group but it must be hollow since every proper subgroup of $Z\left(p^{\infty}\right)$ is finite while the group itself is infinite.

The author would like to thank the referee for pointing out this last example, as well as for other editorial advice.

\section{REFERENCES}

1. P. M. Cohn, Free Rings and Their Relations, London, Academic Press, 1971.

2. P. Fleury, A note on dualizing Goldie dimension, (to appear, Canadian Mathematical Bulletin).

3. A. W. Goldie, Rings with maximum condition, Mimeographed notes, Yale University, Department of Mathematics, 1964.

4. J. Lambek, Lectures on Rings and Modules, Toronto, Blaisdell, 1966.

5. R. Ware, Endomorphims rings of projective modules, Trans. Amer. Math. Soc., 155 (1971), 233-256.

Received September 10, 1973.

State University College

OF ARTS AND SCIENCES

Plattsburgh, New York 



\section{PACIFIC JOURNAL OF MATHEMATICS}

\section{EDITORS}

RICHARD ARENS (Managing Editor)

University of California

Los Angeles, California 90024

\section{J. DUGUNDJI}

Department of Mathematics University of Southern California Los Angeles, California 90007

D. Gilbarg and J. Milgram

Stanford University

Stanford, California 94305

University of Washington
Seattle, Washington 98105

ASSOCIATE EDITORS
E. F, BECKENBACH
B. H. NEUMANN
F. WOLF
K. Yoshida

\section{SUPPORTING INSTITUTIONS}

\author{
UNIVERSITY OF BRITISH COLUMBIA \\ CALIFORNIA INSTITUTE OF TECHNOLOGY \\ UNIVERSITY OF CALIFORNIA \\ MONTANA STATE UNIVERSITY \\ UNIVERSITY OF NEVADA \\ NEW MEXICO STATE UNIVERSITY \\ OREGON STATE UNIVERSITY \\ UNIVERSITY OF OREGON \\ OSAKA UNIVERSITY
}

\author{
UNIVERSITY OF SOUTHERN CALIFORNIA \\ STANFORD UNIVERSITY \\ UNIVERSITY OF TOKYO \\ UNIVERSITY OF UTAH \\ WASHINGTON STATE UNIVERSITY \\ UNIVERSITY OF WASHINGTON \\ * * * * \\ AMERICAN MATHEMATICAL SOCIETY \\ NAVAL WEAPONS CENTER
}

The Supporting Institutions listed above contribute to the cost of publication of this Journal, but they are not owners or publishers and have no responsibility for its content or policies.

Mathematical papers intended for publication in the Pacific Journal of Mathematics should be in typed form or offset-reproduced, (not dittoed), double spaced with large margins. Underline Greek letters in red, German in green, and script in blue. The first paragraph or two must be capable of being used separately as a synopsis of the entire paper. Items of the bibliography should not be cited there unless absolutely necessary, in which case they must be identified by author and Journal, rather than by item number. Manuscripts, in duplicate if possible, may be sent to any one of the four editors. Please classify according to the scheme of Math. Rev. Index to Vol. 39. All other communications to the editors should be addressed to the managing editor, or Elaine Barth, University of California, Los Angeles, California, 90024.

100 reprints are provided free for each article, only if page charges have been substantially paid. Additional copies may be obtained at cost in multiples of 50 .

The Pacific of Journal Mathematics is issued monthly as of January 1966. Regular subscription rate: $\$ 72.00$ a year (6 Vols., 12 issues). Special rate: $\$ 36.00$ a year to individual members of supporting institutions.

Subscriptions, orders for back numbers, and changes of address should be sent to Pacific Journal of Mathematics, 103 Highland Boulevard, Berkeley, California, 94708.

PUBLISHED BY PACIFIC JOURNAL OF MATHEMATICS, A NON-PROFIT CORPORATION

Printed at Kokusai Bunken Insatsusha (International Academic Printing Co., Ltd.), 270, 3-chome Totsuka-cho, Shinjuku-ku, Tokyo 160, Japan.

Copyright (C) 1973 by Pacific Journal of Mathematics Manufactured and first issued in Japan 


\section{Pacific Journal of Mathematics}

\section{Vol. 53, No. $2 \quad$ April, 1974}

Kenneth Abernethy, On characterizing certain classses of first countable spaces by

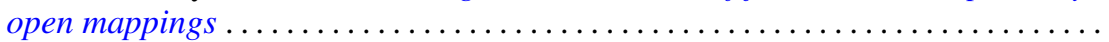

Ross A. Beaumont and Donald Lawver, Strongly semisimple abelian groups .......

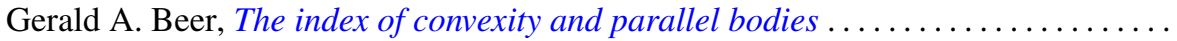

Victor P. Camillo and Kent Ralph Fuller, On Loewy length of rings ..............

Stephen LaVern Campbell, Linear operators for which $T^{*} T$ and $T T^{*}$ commute.

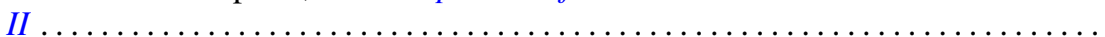

Charles Kam-Tai Chui and Philip Wesley Smith, Characterization of a function by

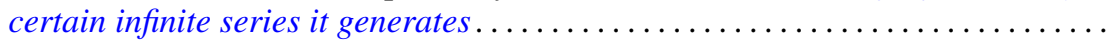

Allan L. Edelson, Conjugations on stably almost complex manifolds . ...........

Patrick John Fleury, Hollow modules and local endomorphism rings . . ..........

Jack Tilden Goodykoontz, Jr., Connectedness im kleinen and local connectedness in

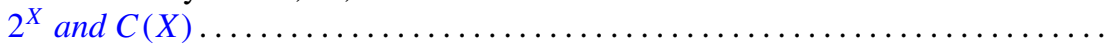

Robert Edward Jamison, II, Functional representation of algebraic intervals .......

Athanassios G. Kartsatos, Nonzero solutions to boundary value problems for

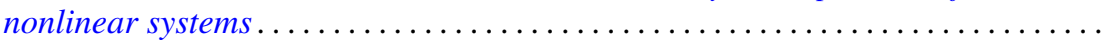

Soon-Kyu Kim, Dennis McGavran and Jingyal Pak, Torus group actions on simply

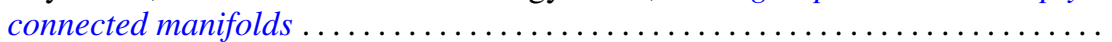

David Anthony Klarner and R. Rado, Arithmetic properties of certain recursively



Ray Alden Kunze, On the Frobenius reciprocity theorem for square-integrable

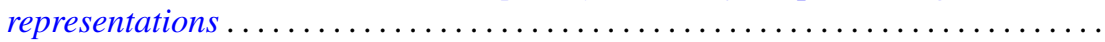

John Lagnese, Existence, uniqueness and limiting behavior of solutions of a class of differential equations in Banach space...

Teck Cheong Lim, A fixed point theorem for families on nonexpansive mappings Lewis Lum, A quasi order characterization of smooth continua

Andy R. Magid, Principal homogeneous spaces and Galois extensions . .

Charles Alan McCarthy, The norm of a certain derivation ..... . .

Louise Elizabeth Moser, On the impossibility of obtaining $S^{2} \times S^{1}$ by elementary surgery along a knot. .

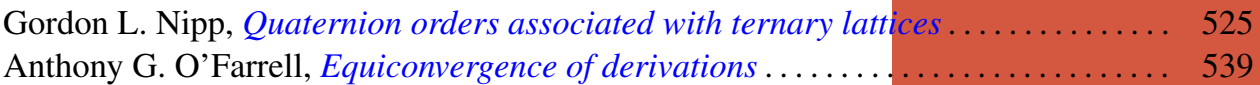

Dorte Olesen, Derivations of $A W^{*}$-algebras are inner . . . . . . . . . . . . . . . 555

Dorte Olesen and Gert Kjærgaard Pedersen, Derivations of $C^{*}$-algebras have

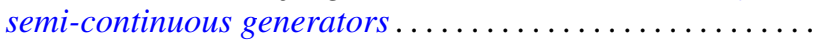

Duane O’Neill, On conjugation cobordism.

Chull Park and S. R. Paranjape, Probabilities of Wiener paths crossing differentiable

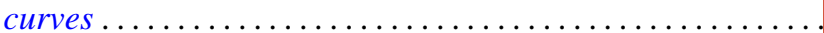

Edward Ralph Rozema, Almost Chebyshev subspaces of $L^{1}(\mu$;

Lesley Millman Sibner and Robert Jules Sibner, A note on the Atiyah-Bott fixed

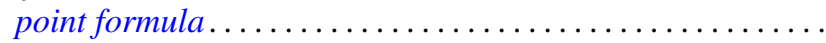

Betty Salzberg Stark, Irreducible subgroups of orthogonal groups generated by

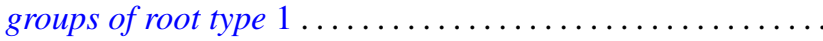

N. Stavrakas, A note on starshaped sets, $(k)$-extreme points and the half ray

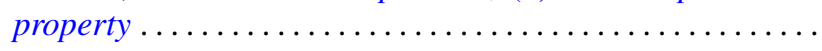

Carl E. Swenson, Direct sum subset decompositions of $Z \ldots \ldots$ 